\title{
Objective in vivo analysis of anti-smoking cigarette filters
}

\author{
NÓIRÍN F SHEAHAN, D PAVIA, J R M BATEMAN, J E AGNEW, AND S W CLARKE \\ From the Departments of Thoracic Medicine and Medical Physics, The Royal Free Hospital, London
}

ABSTRACT Cigarette filters have been introduced to reduce inhaled smoke and also as a means of breaking the smoking habit. Twelve volunteers smoked cigarettes through four ventilated antismoking filters (MD-4, Miles Laboratories) and one reference cigarette without an anti-smoking filter in a single-blind, crossover manner. The amount of smoke inhaled was monitored by a radiotracer technique using the isotope ${ }^{81 \mathrm{~m}} \mathrm{krypton}$. Compared to the reference cigarette the amount of isotope reaching the lung was reduced to $76 \%, 63 \%, 43 \%$, and $37 \%$ for filters 1 to 4 , respectively, which was less than the reduction to $70 \%, 40 \%, 30 \%$, and $20 \%$ predicted by the manufacturers. In the case of filters 2,3 , and 4 , the observed reductions in isotope inhalation were significant $(p \leqslant 0 \cdot 01)$ but were also significantly less $(p \leqslant 0.01)$ than the manufacturers' predictions.

Many cigarette filters have been introduced to reduce inhaled smoke; MD-4 filter packet was introduced in the United Kingdom in 1973 as an aid to stopping smoking. The packet consists of four, staged filters, which are ventilated by a single hole (fig 1), the size of which increases from the first (filter 1) to the fourth (filter 4) filtration to use each filter in turn for two weeks before stopping smoking; thus his nicotine intake falls gradually over two months, so that the pharmacological dependence on the drug is reduced before the physical habit itself is tackled.

The manufacturers' estimate of the efficacy of the filters has been supported by $\mathrm{T} \mathrm{H}$ Houseman

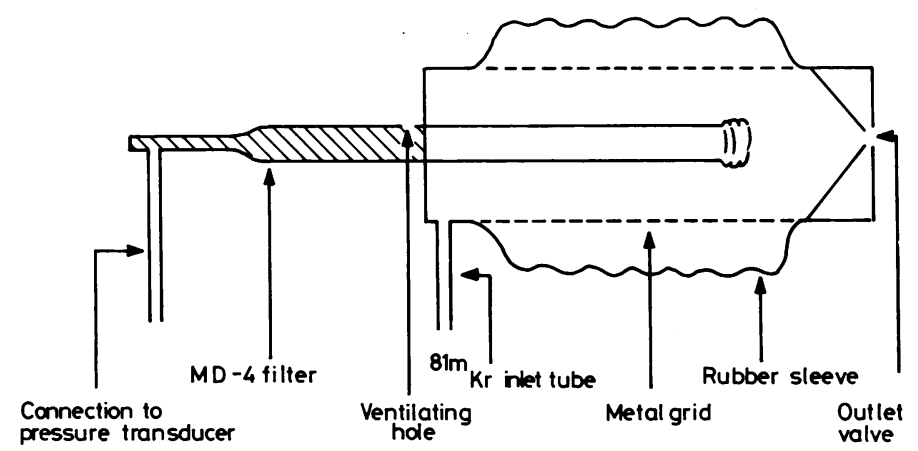

Fig 1 Diagram of cigarette chamber and MD-4 filter assembly.

stage. In all other aspects the filters are identical and contain condensing surfaces to trap tar. The manufacturers estimate that smoke intake may be reduced to $70 \%$ of the normal level using filter 1 , and to $40 \%, 30 \%$, and $20 \%$ using filters 2,3 , and 4 respectively. The purchaser is advised

Address for reprint requests: Miss NF Sheahan, Department of Radiology, Mater Misericordiae Hospital, Dublin 7, Eire. (personal communication from Miles Laboratories) using a machine smoking technique. He also concluded that the reduction in smoke transmission was caused mostly by the ventilation hole rather than the tar traps. The degree of ventilation was similar for a brand of high, medium, and low tar cigarette despite the low resistance to flow offered by the non-tipped high tar cigarette. 
Machine smoking studies have been criticised by Green, ${ }^{1}$ since the machine cannot vary its smoking method between cigarette or filter types, whereas many authors have shown that smokers modify their behaviour to achieve a high nicotine level. ${ }^{2} 3$ This may be done in a number of ways-for example, simply by increasing the number of cigarettes smoked per day. But the more subtle behavioural changes, such as variations in the size of the smoke puff or the degree of inhalation, are difficult to monitor subjectively, and therefore difficult to control, although these determine the amount of smoke reaching the lung. The object of this study was to monitor objectively the amount of smoke entering the lung when smokers were first presented with MD-4 filters.

\section{Methods}

Twelve smokers, whose physical characteristics are given in table 1 , volunteered to take part in the study. The inhalation of smoke was monitored by a technique which has been fully described elsewhere. ${ }^{4}$ In brief, this involved tracing the smoke with the isotope ${ }^{81^{\mathrm{m}}} \mathrm{Kr}$, which has a halflife of $13 \mathrm{~s}$ and emits a gamma-ray of energy $140 \mathrm{keV}$. The cigarette was placed in a chamber (fig 1) in which a constant level of ${ }^{81^{\mathrm{m}}} \mathrm{Kr}$ activity was maintained by continuous injection of the isotope, which was carried in air (in tracer quantities) from the ${ }^{81^{\mathrm{m}}} \mathrm{Kr}$ generator (supplied by MRC Cyclotron Unit, Hammersmith Hospital). The rubber sleeve bordering the chamber could collapse as a puff was taken; a metal grid protected the sleeve from the burning cigarette, and an outlet valve allowed gases to escape from the chamber betwen puffs. The cigarette was attached to the MD-4 filter in the usual way, the latter being held outside the chamber so that air drawn through the ventilation hole to dilute the smoke was not contaminated with activity and the amount of ${ }^{81 \mathrm{~m}} \mathrm{Kr}$ reaching the lung was proportional to the amount of smoke taken from the cigarette. The smoker sat in front of a scintillation detector collimated to view the whole lung field, and the maximal count-rate from the detector obtained during inhalation of a puff of smoke was recorded and corrected for ${ }^{81 \mathrm{~m}} \mathrm{Kr}$ decay; this quantity was termed the "whole lung activity." Since krypton is a poorly soluble, inert gas it washes out of the lung rapidly, and this fact, combined with the short half-life of the isotope, allowed each puff to be examined separately.

The relative puff volume was monitored by connecting the mouthpiece of the filter to a pressure transducer and then integrating the pressure drop during puffing. This system was calibrated for each filter type by taking known volumes of smoke into a syringe and found to be satisfactory for a variety of puff profiles and for the (small) range of tobacco rod lengths used in this study.

Each volunteer took five puffs through each of the MD-4 filters and also through a plain cigarette holder designed to appear similar to an MD-4 filter, in a single-blind crossover manner. The cigarette brand used throughout the study was Benson and Hedges King Size, which in terms of resistance to flow is typical of a wide range of commercially available cigarettes, and a fresh cigarette was substituted each time the filter was changed. The average whole lung activity and puff volume for the five puffs were calculated and then expressed as a percentage of that found when the cigarette holder alone was used; these quantities are referred to hereafter as the "whole lung activity, \%" and the "puff volume, \%," respectively.

Table 1 Physical characteristics of study group

\begin{tabular}{|c|c|c|c|c|c|c|}
\hline Subject & $\operatorname{Sex}$ & Age (yr) & Height $(m)$ & $\begin{array}{l}\% \text { Predicted } \\
F E V_{1}\end{array}$ & $\begin{array}{l}\text { Cigarettes } \\
\text { per day }\end{array}$ & $\begin{array}{l}\text { Total tobacco } \\
\text { consumption } \\
\text { (pack years) }\end{array}$ \\
\hline $\begin{array}{r}1 \\
2 \\
3 \\
4 \\
5 \\
6 \\
7 \\
8 \\
9 \\
10 \\
11 \\
12\end{array}$ & $\begin{array}{l}\mathbf{F} \\
\mathbf{F} \\
\mathbf{M} \\
\mathbf{F} \\
\mathbf{F} \\
\mathbf{F} \\
\mathbf{M} \\
\mathbf{M} \\
\mathbf{M} \\
\mathbf{M} \\
\mathbf{M} \\
\mathbf{M}\end{array}$ & $\begin{array}{l}23 \\
24 \\
31 \\
24 \\
23 \\
39 \\
32 \\
23 \\
24 \\
27 \\
50 \\
21\end{array}$ & $\begin{array}{l}1.63 \\
1.56 \\
1.70 \\
1.59 \\
1.68 \\
1.57 \\
1.68 \\
1.85 \\
1.75 \\
1.80 \\
1.87 \\
1.80\end{array}$ & $\begin{array}{r}107 \\
102 \\
112 \\
105 \\
74 \\
129 \\
106 \\
113 \\
129 \\
107 \\
114 \\
109\end{array}$ & $\begin{array}{l}20 \\
15-20 \\
25 \\
10 \\
20 \\
10-15 \\
60 \\
15 \\
15 \\
15 \\
40 \\
20\end{array}$ & $\begin{array}{c}8 \\
6 \\
12 \cdot 5 \\
2 \\
5 \\
14 \\
33 \\
4 \cdot 5 \\
4 \\
9 \\
35 \\
10\end{array}$ \\
\hline $\begin{array}{l}\text { Mean } \\
\text { SD }\end{array}$ & - & $\begin{array}{r}28 \\
9\end{array}$ & $\begin{array}{l}1 \cdot 71 \\
0 \cdot 11\end{array}$ & $\begin{array}{r}109 \\
14\end{array}$ & $\begin{array}{l}23 \\
14\end{array}$ & $\begin{array}{l}12 \\
11\end{array}$ \\
\hline
\end{tabular}




\section{Results}

The whole lung activity, $\%$, and puff volume, $\%$, for each subject and filter type are given in table 2. Since the numbers involved in the study were small, non-parametric statistics were used throughout for the analysis of the results. Using Wilcoxon's test for pair differences, ${ }^{5}$ the mean reduction in whole lung activity, $\%$, to $76 \%$ in the case of filter 1 was not significant, but neither was this significantly different from the predicted value of $70 \%$. In the case of filters 2,3 , and 4, the mean reductions to $63 \%, 43 \%$, and $37 \%$ respectively, were significant at the $1 \%$ level, but these were also significantly higher $(\mathrm{p} \leqslant 0.01)$ than the levels predicted by the in vitro analysis. There were no changes in puff flow rates but increases to $111 \%$ and $114 \%$ in puff volume for filters 3 and 4 were significant at the $\leqslant 5 \%$ level. nicotine levels of a group of smokers using filters 1 and 2 of the MD-4 series and found these to be similar to the predicted levels for filter 1 , but higher than expected for filter 2. We found no correlation between the degree of ventilation and puff volume or flow rate.

It is interesting to examine the role played by puff volume in determining the whole lung activity: if one assumes the manufacturers' predictions to be correct (in the absence of any smoker compensation), then the small increase in puff volume for filters 3 and 4 could not fully explain the larger deviations in the whole lung activity from the predicted levels. Thus some other form of compensation must have been involved, the most obvious factor being variations in the amount of smoke which leaks from the mouth before inhalation to the lung. This emphasises the importance of monitoring smoke

Table 2 Individual whole lung activity, $\%(W L A \%)$ and puff volume, $\%(P V \%)$ for each filter type

\begin{tabular}{|c|c|c|c|c|c|c|c|c|}
\hline \multirow[t]{2}{*}{ Subject } & \multicolumn{2}{|l|}{ Filter 1} & \multicolumn{2}{|l|}{ Filter 2} & \multicolumn{2}{|l|}{ Filter 3} & \multicolumn{2}{|l|}{ Filter 4} \\
\hline & $\overline{W L A \%}$ & $P V \%$ & $\overline{W L A \%}$ & $P V \%$ & $\overline{W L A \%}$ & $P V \%$ & $\overline{W L A \%}$ & $P V \%$ \\
\hline $\begin{array}{r}1 \\
2 \\
3 \\
4 \\
5 \\
6 \\
7 \\
8 \\
9 \\
10 \\
11 \\
12\end{array}$ & $\begin{array}{r}63 \\
80 \\
56 \\
47 \\
130 \\
51 \\
144 \\
64 \\
62 \\
72 \\
74 \\
74\end{array}$ & $\begin{array}{r}142 \\
79 \\
95 \\
93 \\
97 \\
70 \\
110 \\
106 \\
136 \\
103 \\
114 \\
89\end{array}$ & $\begin{array}{r}53 \\
63 \\
49 \\
41 \\
90 \\
57 \\
124 \\
52 \\
45 \\
61 \\
47 \\
69\end{array}$ & $\begin{array}{r}75 \\
71 \\
95 \\
89 \\
103 \\
42 \\
116 \\
97 \\
116 \\
99 \\
108 \\
86\end{array}$ & $\begin{array}{l}40 \\
41 \\
38 \\
36 \\
60 \\
48 \\
61 \\
37 \\
37 \\
39 \\
40 \\
34\end{array}$ & $\begin{array}{r}125 \\
93 \\
109 \\
80 \\
128 \\
79 \\
114 \\
116 \\
127 \\
129 \\
123 \\
88\end{array}$ & $\begin{array}{l}33 \\
36 \\
27 \\
23 \\
75 \\
30 \\
65 \\
33 \\
38 \\
34 \\
28 \\
24\end{array}$ & $\begin{array}{r}131 \\
79 \\
107 \\
84 \\
146 \\
84 \\
118 \\
127 \\
139 \\
126 \\
108 \\
109\end{array}$ \\
\hline Mean & 76 & 103 & 63 & 91 & 43 & 109 & 37 & 113 \\
\hline SE & 8 & 5 & 7 & 5 & 3 & 4 & 5 & 5 \\
\hline
\end{tabular}

\section{Discussion}

The fact that no significant reduction in whole lung activity was seen for filter 1 resulted from two subjects inhaling far more smoke when using the filter than without it. However, the group as a whole showed no significant deviation from the predicted result (fig 2), indicating that dilution to $70 \%$ is acceptable to many, though not all, smokers. For filters 2, 3, and 4 the reduction in whole lung activity was highly significant, since almost all smokers inhaled considerably less smoke using these filters. However, subjects also inhaled more than the amount predicted by the in vitro analysis, so that some degree of compensation may be expected (at least initially) when filters diluting smoke to $\leqslant 40 \%$ are used. These findings support the work of Sutton, ${ }^{6}$ who monitored carboxyhaemoglobin and plasma

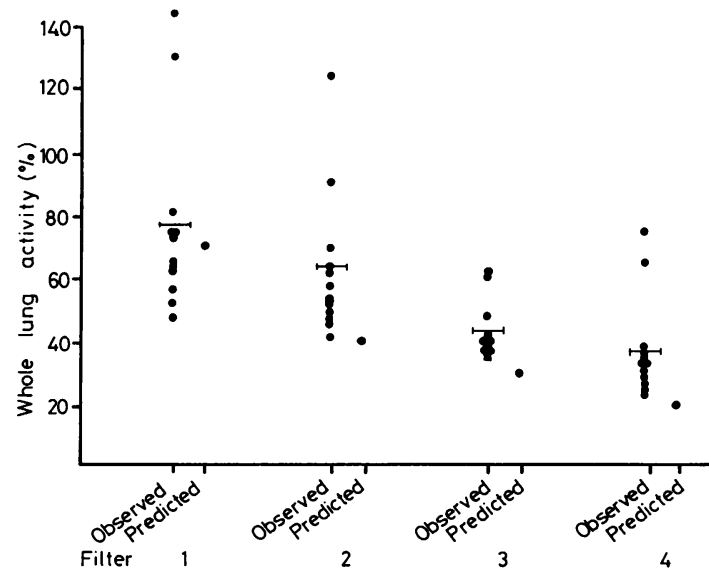

Fig 2 Comparison of observed and predicted whole lung activity, \%. 
intake to the lung, as opposed to the mouth, in all smoking behavioural studies. ${ }^{81 \mathrm{~m}} \mathrm{Kr}$, which is available from a portable generator system, ${ }^{7}$ is a very convenient tracer for this purpose.

In conclusion, smoke intake was reduced by using filters 2,3 , and 4 , but the reductions were not as great as the manufacturers predicted. Since certain smokers inhaled more smoke while using filter 1 than without an MD-4 filter, no significant reduction in smoke intake was noted for this filter in the population studied.

We would like to thank Miles Laboratories for providing financial assistance for this project.

\section{References}

1 Green SJ. Ranking cigarette brands on smoke deliveries. In: Thornton RE, ed. Smoking behaviour: physiological and psychological influences. Edinburgh: Churchill Livingstone, 1978: $380-8$.

2 Rawbone RG, Murphy K, Tate ME, Kane SJ. The analysis of smoking parameters, inhalation and absorption of tobacco smoke in studies of human smoking behaviour. In: Thornton RE, ed. Smoking behaviour: physiological and psychological influences. Edinburgh: Churchill Livingstone, 1978:171-94.

3 Ashton H, Watson DW. Puffing frequency and nicotine intake in cigarette smokers. $\mathrm{Br}$ Med $\mathrm{J}$ 1970; 3:679-81.

4 Sheahan NF, Pavia D, Bateman JRM, Agnew JE, Clarke SW. A technique for monitoring the inhalation of cigarette smoke in man using krypton-81m. Int J Appl Radiat Isotopes 1980; 31:438-41.

5 Diem K. Documenta Geigy: scientific tables. Sixth edition. Manchester: Geigy Pharmaceutical Company, 1968:128.

6 Sutton SR, Russell MAH, Feyerabend C, Saloojee Y. Smokers response to dilution of smoke by ventilated cigarette holders. In: Thornton RE, ed. Smoking behaviour: physiological and psychological influences. Edinburgh: Churchill Livingstone, 1978:330-5.

7 Clark JC, Horlock PL, Watson IA. Krypton $81 \mathrm{~m}$ generators for ventilation and perfusion. In: Lavender JP, ed. Clinical and experimental applications of krypton $81 \mathrm{~m}$. British Journal of Radiology Special Report No 15. London: British Institute of Radiology, 1978:7-15. 